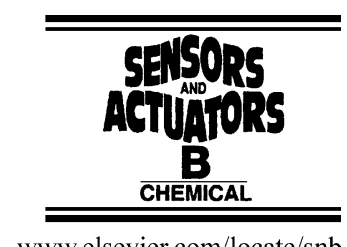

www.elsevier.com/locate/snb

\title{
A novel real-time PCR machine with a miniature spectrometer for fluorescence sensing in a micro liter volume glass capillary
}

\author{
Da-Sheng Lee ${ }^{\mathrm{a}, *}$, Meng-Hsun Wu ${ }^{\mathrm{a}}$, U. Ramesh ${ }^{\mathrm{a}}$, Chien-Wei Lin ${ }^{\mathrm{b}}$, \\ Tzu-Mei Lee ${ }^{b}$, Ping-Hei Chen ${ }^{a}$ \\ a Mechanical Engineering Department, National Taiwan University, Taipei 10617, Taiwan, ROC \\ b General Biologicals Corp., Hsinchu, Taiwan, ROC
}

Received 22 September 2003; received in revised form 16 January 2004; accepted 5 February 2004

Available online 28 March 2004

\begin{abstract}
This study sets up a prototype of real-time polymerase chain reaction (RT-PCR) machine that employs a miniature spectrometer for detecting the emission of fluorescence intensity from RT-PCR mix in a micro liter volume glass capillary. The RT-PCR machine is one of the major instruments for SARS virus test during the outbreak in Asia in early 2003. Comparing with traditional RT-PCR machine with discrete channels fluorescence wavelength detection, the prototype can provide continuous wavelength detection and can be employed for multiplex DNA quantification. However, only one HBV SC 11 DNA template with the SYBR Green I labeling dye were used in this study to compare DNA quantification accuracy and reproducibility of the present system and the commercial system. The two machines have different optical engine arrangement and so two separate analytical models were proposed to predict the fluorescence intensity from the RT-PCR mix during thermal cycling for the machines. Predicted results agree well with the measured data for both machines. From the predicted results, different approaches should be adopted to determine the initial DNA concentration for quantification from time recorded history of the fluorescence intensity. Measured results illustrate that the RT-PCR prototype has the same accuracy for DNA quantification and reproducibility within five intra-assay samples as compared with the commercial machine.
\end{abstract}

(C) 2004 Elsevier B.V. All rights reserved.

Keywords: RT-PCR; Spectrometer; Discrete channels fluorescence detection; Continuous wavelength detection

\section{Introduction}

Mullis et al. [1] developed polymerase chain reaction (PCR) in 1986 to duplicate specified DNA fragments in vitro through temperature cycling. Since then, PCR becomes one of the major tools in genomic studies. By integrating a fluorometer with a temperature cycler, a real-time PCR (RTPCR) machine was first introduced in 1997 [2,3]. Fig. 1 shows the process by which the fluorescence intensity of labeling dye in PCR mix increases with the number of specific double strand DNA fragments that are duplicated in thermal cycling. This RT-PCR system allows detection of DNA amplification through the detection of fluorescence labeling dye in the PCR mix during the early phase of reaction. In addition, the concentration of target DNA fragment in the PCR mix before thermal cycling can be obtained from the recorded time history of fluorescence intensity. The RTPCR machine has high sensitivity and consumes less time

\footnotetext{
* Corresponding author.
}

when compared to traditional PCR machine. The RT-PCR machine can detect two fold amplification of DNA template while traditional PCR can only detect the signal after 10 fold amplification of DNA template [4]. In addition, after thermal cycling from the RT-PCR machine, there is no need to perform agarose gel electrophoresis on PCR mix, while this is inevitable for amplified PCR mix from the traditional PCR machine to ensure a successful amplification on DNA template.

The current commercial RT-PCR machine has three discrete channels with photodiodes for detecting fluorescence emitted from PCR mix. In order to separate different fluorescence wavelengths, one set of narrow band filters and dichrotic mirrors are used to allow fluorescence with the required wavelength to reach the corresponding channel. Since the emitting dye fluorescence has to pass through complicated optical path before it reaches the appropriate channel, the intensity is decayed to $40-50 \%$ of the fluorescence intensity detected by the previous channels [5]. Therefore, it is impossible to have five different dye detection capabilities in one sample of PCR mix due to optical loss. However, 


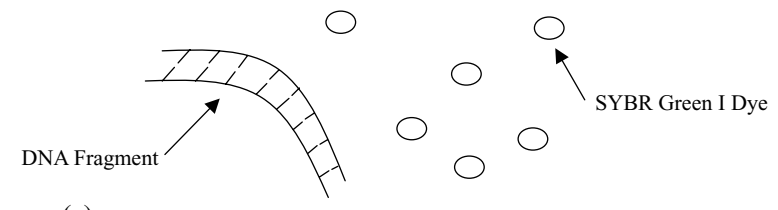

(a)

(b)

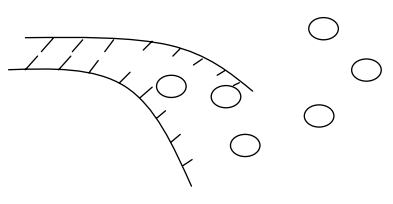

(c)
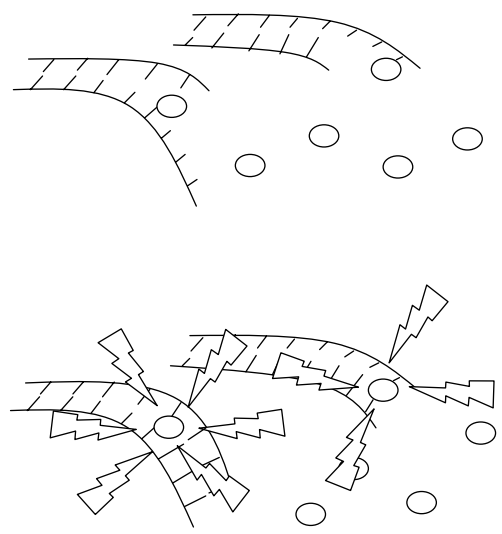

(d)

Fig. 1. Increase of fluorescence intensity of SYBR Green I dye with amplification of specific double strand DNA fragment. (a) Floating SYBR Green I dye with double strand DNA fragment in the PCR mix. (b) When DNA fragment is denatured, SYBR Green I dye is attached to single strand DNA. (c) Single strand DNA fragment is duplicated by the polymerase. (d) When DNA amplification is complete, SYBR Green I dye binds to the double strand DNA, emitting fluorescence as it is excited.

simultaneous detection of multiple target DNA sequence becomes a trend in either virus or blood inspection. It is also useful to detect HBV, HCV, HIV, and other virus in one blood sample. Therefore, the present study proposes a miniature spectrometer to replace the limited number of detection channels by photodiodes. This spectrometer is allowed to detect a continuous spectrum of fluorescence. Therefore, more labeling dyes can be used in PCR mix for RT-PCR machine with the spectrometer than with three photo sensors as in the commercial RT-PCR machine.

Traditional spectrometer with concave holographic grating has only $25-35 \%$ efficiency between fluorescence wavelength of $300-800 \mathrm{~nm}$. This makes it necessary to cool the detector to $-70^{\circ} \mathrm{C}$ in order to achieve high sensitivity on spectral distribution of emitted light. Thus, the whole system with traditional spectrometer is too big and expensive for commercial instrument.

Instead of concave grating, a new cylindrical grating was introduced [6]. Its detection efficiency can reach a value of $70 \%$. By employing the novel spectrometer, a prototype for RT-PCR machine was constructed and tested. To validate the performance of the prototype, single DNA template, HBV SC 11, with known concentrations and a single labeling dye, SYBR Green I, were used in PCR mix that went through the same thermal cycling in both the prototype and the commercial RT-PCR machine. Comparisons were made for all measurements obtained from both machines. Additionally, two separate models are proposed to predict fluorescence intensity emitted from glass capillary during the thermal cycling of PCR mix. Both measured and predicted fluorescence intensities of PCR mix during thermal cycling in both machines are presented.

\section{Experimental apparatus}

In this study, the Light Cycler-Fast Start DNA master with Cat no. 3003230 and SYBR Green I labeling dye was employed as the PCR mix for quantification of HBV SC 11 DNA fragment with different initial DNA copies. The test sample was loaded into a micro liter volume capillary and sealed with a plastic plug. The capillary has an inner diameter of $0.8 \mathrm{~mm}$ and an outer diameter of $1.15 \mathrm{~mm}$. The composite plastic/glass container for both the present prototype and the commercial PCR machine are shown in Fig. 2.

The DNA sample capillary tube was loaded on a circular carousel and inserted into the cylindrical temperature control chamber. Temperature control is done by air flow through a wind duct with a $300 \mathrm{~W}$ heater. For heating, the heater is proportionally controlled and the fan on top of the wind duct is run at a low speed. For cooling, the heater power will be dropped and the fan will be run at high speed. The
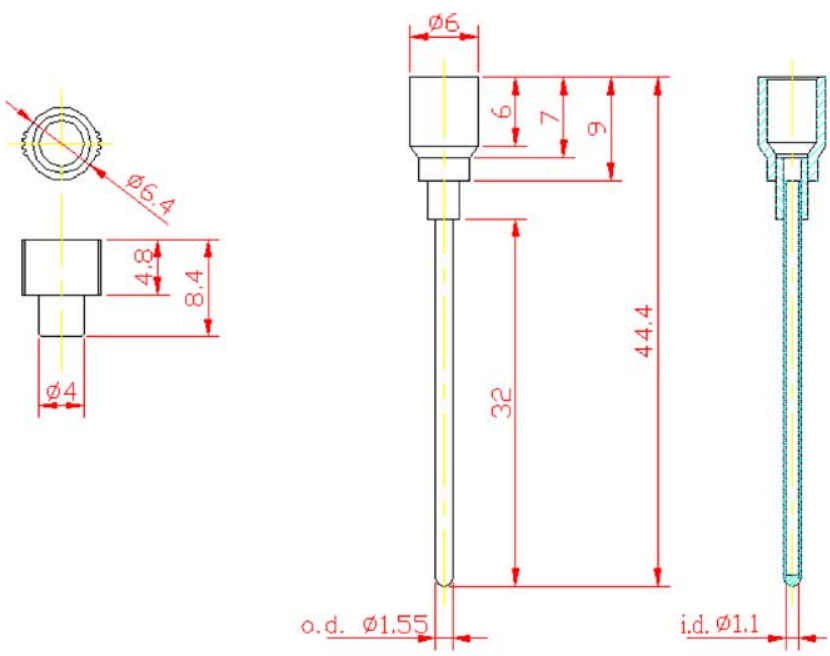

(a)

(b)

Fig. 2. A micro liter volume glass capillary for PCR mix (a) plastic cap (b) main body. 
machine will run thermal cycle to duplicate DNA template and record the fluorescence intensity to determine initial DNA copies. The thermal cycling control structure is similar to that of the commercial RT-PCR machine with discrete channels of fluorescence detection. Both machines employ the same thermal cycling condition of $10 \mathrm{~min}$ incubation at $95^{\circ} \mathrm{C}$, each cycle of $3 \mathrm{~s}$ of $95^{\circ} \mathrm{C}$ denaturation, $10 \mathrm{~s}$ of $53^{\circ} \mathrm{C}$ annealing, and $16 \mathrm{~s}$ of $72{ }^{\circ} \mathrm{C}$ elongation. The sample temperature can be maintained precisely with a variation of $0.4{ }^{\circ} \mathrm{C}$ at $94{ }^{\circ} \mathrm{C}$, a variation of $0.2^{\circ} \mathrm{C}$ at $56^{\circ} \mathrm{C}$, and a variation of $0.2^{\circ} \mathrm{C}$ at $72{ }^{\circ} \mathrm{C}$.

The RT-PCR fluorescence sensing optical engine is designed for recording fluorescence accumulation in the PCR reaction stage and there is a quantitative relationship between the amount of starting point of DNA fragment and the amount of amplified product at the given temperature cycle number. Accurate results of DNA quantification can be obtained. The major difference between the two RT-PCR machines is the fluorescence sensing system. Both commercial and prototype RT-PCR machines are tested in this study and the DNA quantification accuracy and reproducibility are the system performance index for comparison. The fluorescence sensing system of the two machines will be introduced in the following section.

\subsection{Fluorescence sensing system of the commercial RT-PCR machine}

The commercial machine (Roche, Light Cycler, USA) is widely employed for DNA quantification. The optical engine design with three discrete fluorescence detection channels is shown in Fig. 3.

The design is based on division of fluorescence emitted from PCR mix into three channels by the beam split mirrors and filters array. The first channel employs $575 \mathrm{~nm}$ beam splitter for dividing fluorescence, and interference band pass filter at 530 and $40 \mathrm{~nm}$ bandwidth to detect fluores-

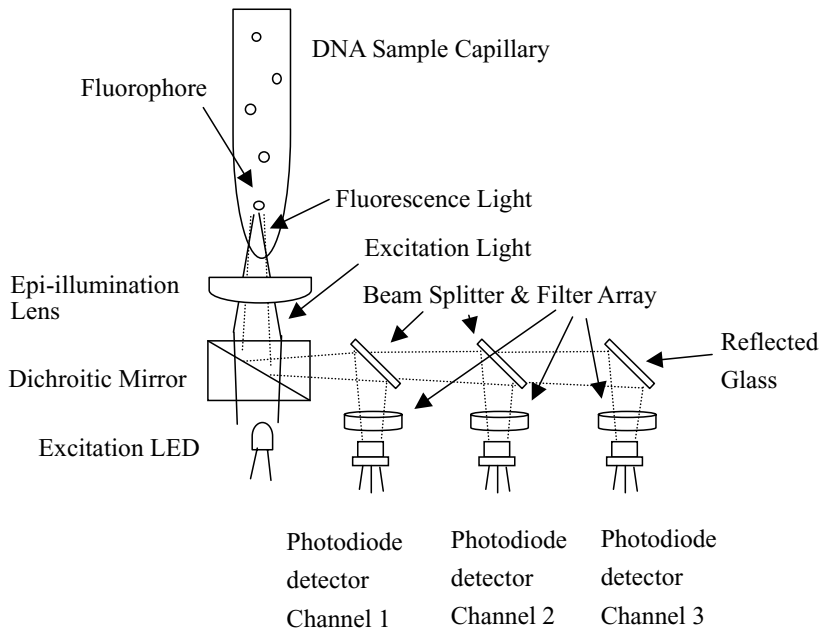

Fig. 3. A schematic view of fluorescence sensing system of commercial RT-PCR machine. cence emission such as SYBR Green dye. The other two channels employ $670 \mathrm{~nm}$ beam splitter and reflect mirror for dividing fluorescence, and interference filters with 645 and $710 \mathrm{~nm}$, and 20 and $30 \mathrm{~nm}$ bandwidths, respectively, to detect the long wavelength fluorescence emission such as LightCycler-Red 640 and LightCycler-Red 705. The SYBR Green dye can be filtered by the filtered exciting light from the blue light emitting from diode filtered by $470 \mathrm{~nm}$ band pass filter. By fluorescence resonance energy transfer, Red 640 and Red 750 can also be excited by the same light source.

This kind of structure has an advantage of relatively inexpensive cost and, the filters and excitation source are modularized to allow real-time fluorescence analysis. However, the collection efficiency of dye fluorescence that is directed to the appropriate channel is $40-50 \%$ even in case of ideal filter performance. Beyond five channels, the optical loss will exceed two orders. This limits DNA quantification capability for multiplex PCR analysis.

\subsection{Continuous fluorescence wavelength sensing system of the prototype}

The novel design employs the spectrometer for continuous fluorescence spectral dispersion. All the collected fluorescence light is directed towards the slit detector and there is no loss from multiple beam splitters and filters. The detection fluorescence wavelength ranges from 350 to $1100 \mathrm{~nm}$ with $10 \mathrm{~nm}$ discrepancy and no channel number limitation for the system.

RT-PCR with spectral dispersion detection of fluorescence is shown schematically in Fig. 4. Since the feature of this system is multi-labeling-dye detection, the optical engine can be modified to provide excitation light with continuous spectral output, and the appropriate wavelength excitation light separated by the sliding filters array can excite the multi-fluorophores in the DNA sample capillary through the side. The fluorescence emission collected by focusing lens transports through the optical fiber. Fluorescence light from the optical fiber enters the optical engine through the fiber connector. One collimating mirror reflects the light, as a collimated beam, towards the grating. The cylindrical grating can disperse the light in the spectral range from 350 to $1100 \mathrm{~nm}$ onto different positions of the linear CCD array and the multi-labeling-dye fluorescence with different wavelengths can be detected.

Fig. 5 shows the system prototype. A $35-\mathrm{W}$ power output Xenon bulb (Ocean Optics Inc., HPX-2000-B, USA) supplies a continuous spectrum from 350 to $800 \mathrm{~nm}$ for fluorescence excitation. In this study, DNA labeling was done by SYBR Green I dye and only $494 \mathrm{~nm}$ excitation light is needed. Subsequently, the prototype has only one fixed interference band pass filter with central pass wavelength 490 nm (UNICE E-O Service Inc., 490.0-F10-12.7, TWN) and the multi-selection filters array will be assembled in future development. The fluorescence light is excited from 


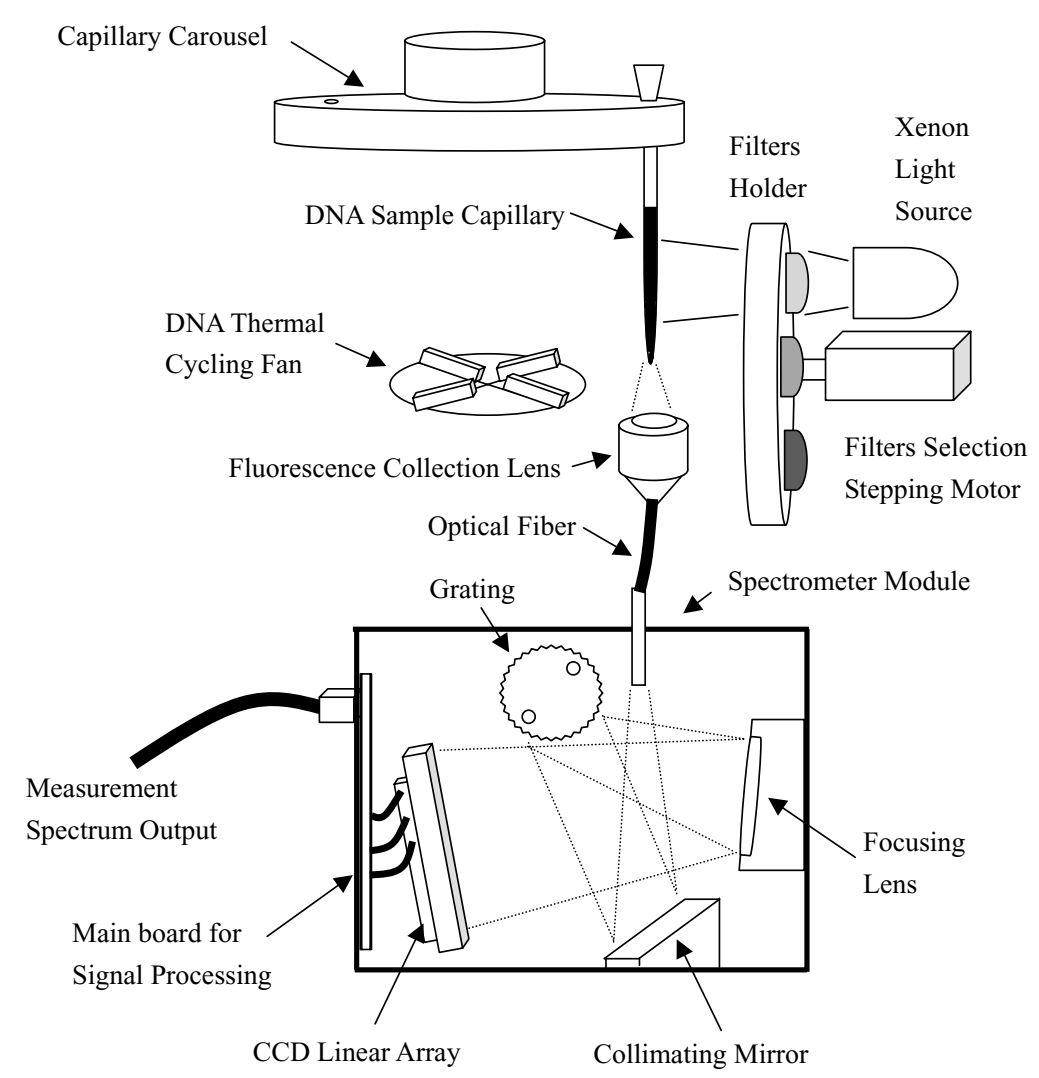

Fig. 4. A schematic view of the prototype with a spectrometer for continuous fluorescence wavelength sensing.

the side of the DNA sample capillary tube and transported through optical fiber linking the focusing lens on the bottom of the DNA sample capillary chamber to the spectrometer. One miniature spectrometer with integrated grating and lens module (Ocean Optics Inc., USB2000-FLG, USA) employed for fluorescence dispersion and CCD linear array (Sony, ILX 511, JPN) responds to the different fluorescence wavelength on each pixel. The main board designed by our lab processes the CCD signal into complete spectrum and translates the data to the user interface software on computer. The thermal cycling control is also built in the same board.

\section{Model for predicting fluorescence intensity}

Two different optical engine designs for RT-PCR fluorescence accumulation monitoring within PCR cycles are introduced. The numerical model is developed to predict the fluorescence signal being the function of DNA concentration within each PCR cycle with respect to the two optical engine designs. The model prediction values can be compared with the commercial discrete detection channels reading, and thus, can prove the validity. The novel design with continuous wavelength detection can employ the model as a basis for analyzing the fluorescence signal measured by the system prototype to achieve the precise RT-PCR quantification.
The most general approximation of the fluorescence modeling yields the following:

$$
\begin{aligned}
\Delta F= & I_{\lambda}(0) \int_{0}^{L} \int_{0}^{2 \pi} \int_{\varphi_{1}(x, \theta, \varphi)}^{\varphi_{2}(x, \theta, \varphi)} \sin \varphi \sum_{i=1}^{M} \phi_{i} a_{i, \lambda} c_{i} \\
& \times\left(\frac{I_{\lambda}(x)}{I_{\lambda}(0)}\right)\left(\frac{I_{\lambda^{\prime}}(L)}{I_{\lambda^{\prime}}(0)}\right) \mathrm{d} \varphi \mathrm{d} \theta \mathrm{d} x .
\end{aligned}
$$

where $\Delta F$ means the fluorescence signal intensity and is proportional to $I_{\lambda}(0)$, excitation light intensity, times the labeling dye quantum yield $\phi_{i}$, the molar extinction coefficient $a_{i, \lambda}$ and the DNA template concentration $c_{i}$ within each PCR cycle $i$. The two terms $\left(I_{\lambda}(x) / I_{\lambda}(0)\right)$ and $\left(I_{\lambda^{\prime}}(L) / I_{\lambda^{\prime}}(0)\right)$ denote the excitation light decay due to absorption of labeling dye and emission fluorescence decay as light passes through the DNA sample capillary tube of length $L$. The integration is along the DNA sample capillary tube length and around the whole core angle $\theta$ by $2 \pi$ and the solid angle $\varphi$ from $\varphi_{1}$ to $\varphi_{2}$ which is determined by the different optical engine arrangements. The summation $M$ is dependent on the number of DNA labeling dyes in the PCR reagent.

\subsection{Predicted fluorescence intensity for commercial RT-PCR machine}

Fig. 6 shows the core angle, the solid angle and the integration length definition of the commercial RT-PCR 


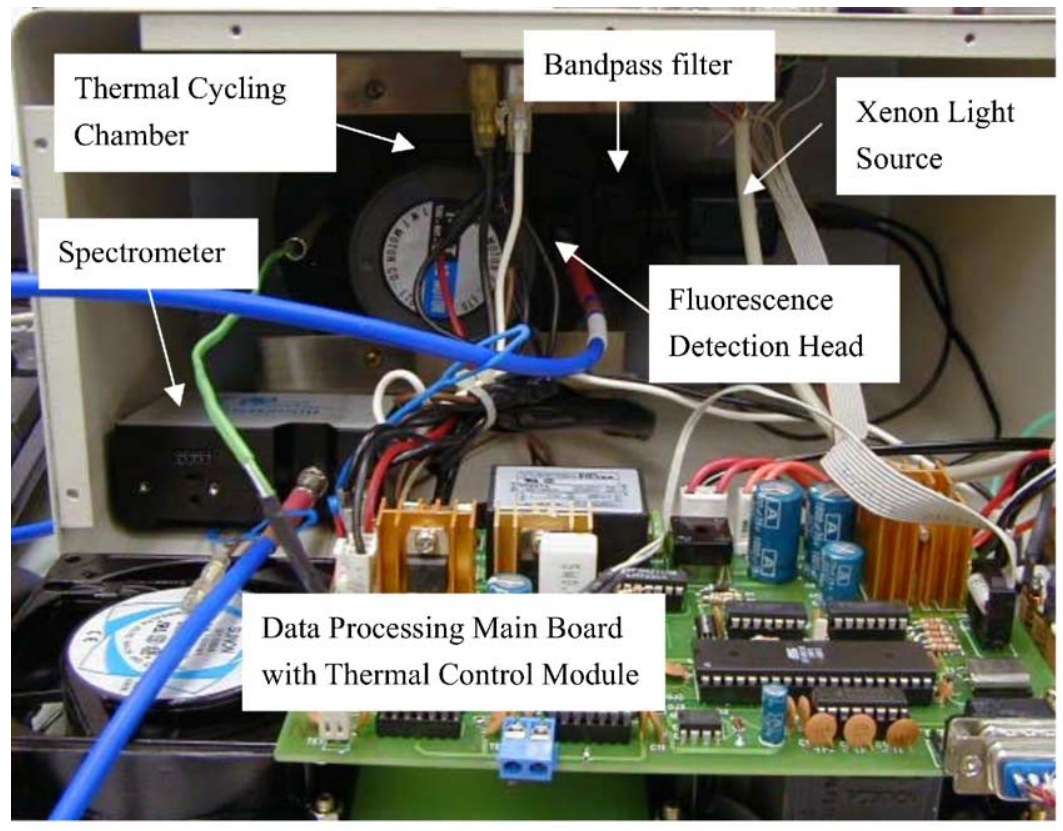

(a)

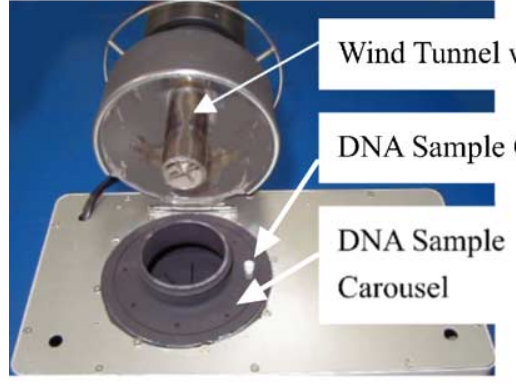

(b)

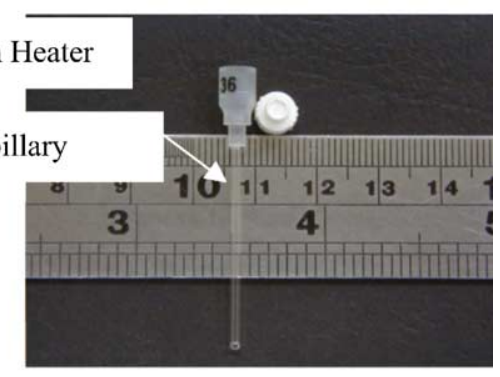

(c)

Fig. 5. The prototype of the RT-PCR machine with continuous fluorescence wavelength detection. (a) The structure of the optical engine, miniature spectrometer, and signal processing system. (b) The top view of the machine prototype. (c) The micro liter volumes DNA sample capillary tube.

machine optical engine. According to the schematic draw-

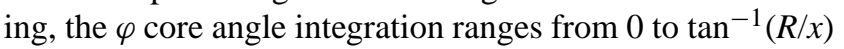
where $R$ is the radius of the epi-illumination lens. In this study, $R$ is $1.8 \mathrm{~cm}$. Eq. (1) can be reduced to

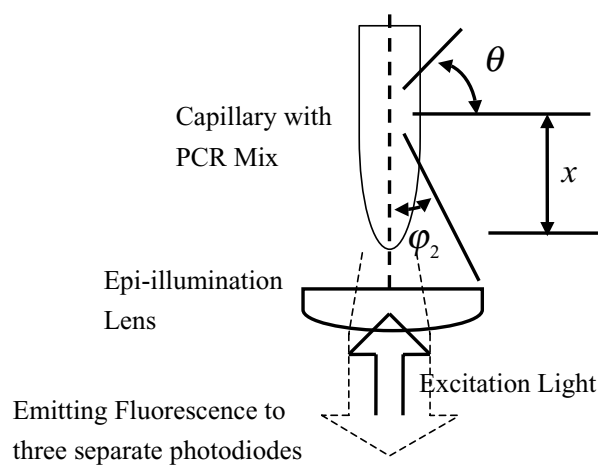

Fig. 6. Definitions of the core angle, the solid angle, and the integration length for the commercial RT-PCR machine with a con-focal excitation.

$$
\begin{aligned}
\Delta F= & 2 \pi I_{\lambda}(0) \int_{0}^{L} \int_{0}^{\tan ^{-1}(R / x)} \sin \varphi \sum_{i=1}^{M} \phi_{i} a_{i, \lambda} c_{i} \\
& \times\left(\frac{I_{\lambda}(x)}{I_{\lambda}(0)}\right)\left(\frac{I_{\lambda^{\prime}}(L)}{I_{\lambda^{\prime}}(0)}\right) \mathrm{d} \varphi^{\prime} \mathrm{d} x .
\end{aligned}
$$

In this study, only one fluorophore SYBR Green I is employed to DNA labeling. The summation $M$ equals to 1 .

The term $\left(I_{\lambda}(x) / I_{\lambda}(0)\right)$ can be estimated by Beer-Lambert law and be expressed as

$$
\left(\frac{I_{\lambda}(x)}{I_{\lambda}(0)}\right)=\exp \left(-x a_{i, \lambda} c_{i}\right)
$$

The decay term $\left(I_{\lambda^{\prime}}(L) / I_{\lambda^{\prime}}(0)\right)$ is due to three factors which include the emission light divergence by the DNA sample vessel, the light absorption by DNA sample solution, and the Raman scattering effect. The divergence term means only a fraction of the emission light is aimed back to the detector because of the refraction of the DNA sample solution and vessel glass wall, as shown in Fig. 7. 


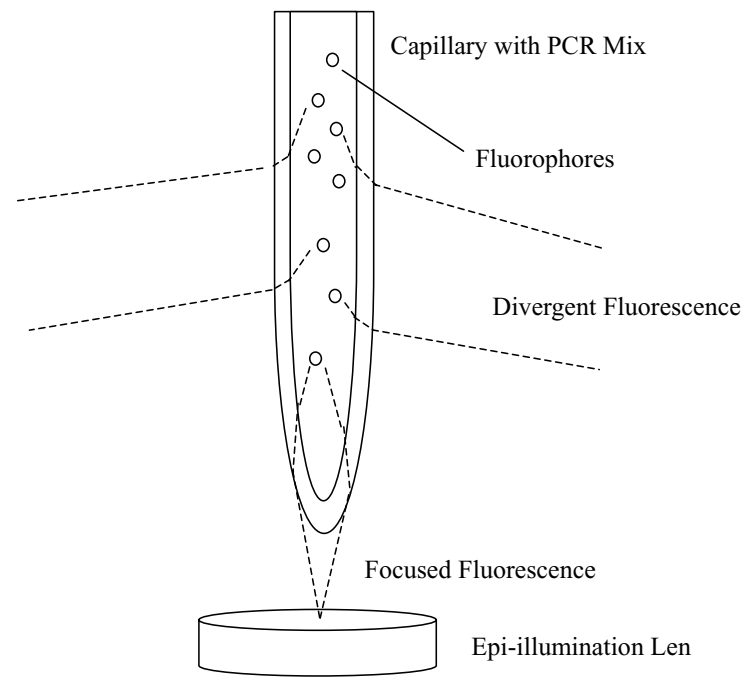

Fig. 7. Fluorescence diffraction due to capillary glass wall.

The decay term can be estimated by

$$
\left(\frac{I_{\lambda^{\prime}}(L)}{I_{\lambda^{\prime}}(0)}\right)=\exp \left(-\frac{L}{r}\right) \text {. }
$$

where $r$ is the radius of the DNA sample capillary.

The value of the optical absorption coefficient can be taken to be the following number from the database of reference [7] and the decay term can be estimated as

$$
\left(\frac{I_{\lambda^{\prime}}(L)}{I_{\lambda^{\prime}}(0)}\right)=\exp (0.000498 L) \text {. }
$$

The Raman scattering coefficient can be referred to reference [8] and be expressed as

$$
\left(\frac{I_{\lambda^{\prime}}(L)}{I_{\lambda^{\prime}}(0)}\right)=\exp \left(3 \times 10^{-9} L\right) \text {. }
$$

Comparing Eqs. (4), (5) and (6), the coefficient of Eq. (4) is 1.2903 since the focusing radius of the DNA capillary is $0.775 \mathrm{~mm}$, and this value is much larger than that of Eqs. (5) and (6). The fluorescence decay term can then be sufficiently represented by only one term, i.e. by Eq. (4).

Substitute Eq. (4) into Eq. (2), the emitting fluorescence intensity of SYBR Green I dye can be calculated by the following integration:

$$
\begin{aligned}
\Delta F= & 2 \pi I_{\lambda}(0) \int_{0}^{L} \int_{0}^{\tan ^{-1}(R / x)} \\
& \times \sin \varphi \phi_{i} a_{i, \lambda} c_{i} \mathrm{e}^{-x a_{i, \lambda} c_{i}} \mathrm{e}^{-l_{2} / r} \mathrm{~d} \varphi \mathrm{d} x .
\end{aligned}
$$

where $\phi_{i}$ is the fluorophore quantum yield efficient constant, and the emission light decay is also constant, and is thus, regardless to the integration. A dimensionless signal gain can be defined as the ratio of the fluorescence signal, $\Delta F$, to the excitation light, $I_{\lambda}(0)$. Eq. (7) can be rewritten as

$$
\frac{\Delta F}{I_{\lambda}(0)}=2 \pi \phi_{i} \mathrm{e}^{-L / r} \int_{0}^{L} \int_{0}^{\tan ^{-1}(R / x)} \sin \varphi a_{i, \lambda} c_{i} \mathrm{e}^{-x a_{i, \lambda} c_{i}} \mathrm{~d} \varphi \mathrm{d} x .
$$

The inner integral can be expressed as the following:

$$
\int_{0}^{\tan ^{-1}(R / x)} \sin \varphi^{\prime} \cdots \mathrm{d} \varphi^{\prime}=\left(1-\frac{x}{\sqrt{x^{2}+R^{2}}}\right) .
$$

Substitute Eq. (9) into Eq. (8), it yields

$$
\frac{\Delta F}{I_{\lambda}(0)}=2 \pi \phi_{i} \mathrm{e}^{-L / r} \int_{0}^{L} a_{i, \lambda} c_{i}\left(1-\frac{x}{\sqrt{x^{2}+R^{2}}}\right) \mathrm{e}^{-x a_{i, \lambda} c_{i}} \mathrm{~d} x .
$$

By incorporating the transition parameter $S$ with a unit of $\mathrm{cm}^{-1}$ as

$S=-\left\{\frac{\log \left(1-x /\left(\sqrt{x^{2}+R^{2}}\right)\right)}{x}\right\}$.

Eq. (10) becomes

$\frac{\Delta F}{I_{\lambda}(0)}=2 \pi \phi_{i} \mathrm{e}^{-L / r} \int_{0}^{L} a_{i, \lambda} c_{i} \mathrm{e}^{-x\left(a_{i, \lambda} c_{i}+S\right)} \mathrm{d} x$.

In this study, $S$ changes little with $x$ as $x$ varies from 0.05 to $2.0 \mathrm{~cm}$. Thus, $S$ can be treated as a constant in Eq. (12) and is given by

$$
\frac{\Delta F}{I_{\lambda}(0)}=\frac{2 \pi \phi_{i} \mathrm{e}^{-L / r_{2}} a_{i, \lambda} c_{i}\left[-\mathrm{e}^{-L\left(a_{i, \lambda} c_{i}+S\right)}+1\right]}{a_{i, \lambda} c_{i}+S} .
$$

By the numerical expression, the fluorescence intensity with respect to excitation light can be predicted within each PCR cycles with different initial DNA template concentration in the sample capillary.

\subsection{Predicted fluorescence intensity for the prototype}

The core angle, the solid angle and the integration length of the RT-PCR prototype with continuous fluorescence wavelength sensing system can be referred to in Fig. 8 . The collimated excitation light directly excites the capillary with PCR mix through the side. The focusing effect by the capillary wall and the short pass length can yield the simple estimation of $I_{\lambda}(0)=I_{\lambda}(d)$, where $d$ denotes the diameter of the capillary.

The integration can be expressed as

$$
\begin{aligned}
\Delta F= & 2 \pi I_{\lambda}(0) \int_{0}^{L} \int_{0}^{\tan ^{-1}(x / R)} \\
& \times \sin \varphi^{\prime} \phi_{i} a_{i, \lambda} c_{i}\left(\frac{I_{\lambda^{\prime}}(L)}{I_{\lambda^{\prime}}(0)}\right) \mathrm{d} \varphi^{\prime} \mathrm{d} x .
\end{aligned}
$$




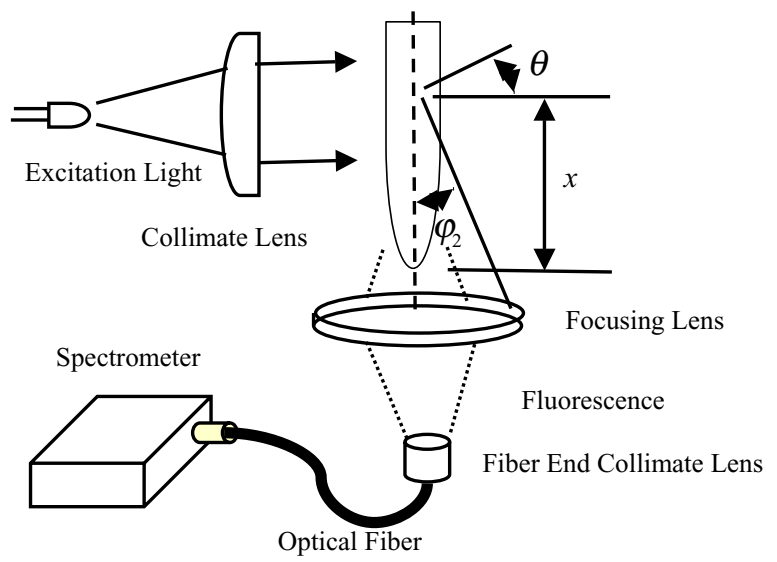

Fig. 8. Definitions of the core angle, the solid angle, and the integration length for the prototype with a side excitation.

The same transition parameter $S$ can be employed for evaluating the integral and thus

$\Delta F=2 \pi I_{\lambda}(0) \phi_{i} a_{i, \lambda} \mathrm{e}^{-L / r} \int_{0}^{L}\left(1-\frac{x}{\sqrt{x^{2}+R^{2}}}\right) c_{i} \mathrm{~d} x$.

Eq. (15) becomes

$\frac{\Delta F}{I_{\lambda}(0)}=2 \pi \phi_{i} a_{i, \lambda} \mathrm{e}^{-L / r} \frac{1}{S}\left(1+\mathrm{e}^{-S L}\right) c_{i}$.

By the formula, the fluorescence intensity with respect to excitation light for the sample with different initial DNA template copies can be predicted.

\section{Determination of initial DNA fragment copies in PCR mix}

For RT-PCR fluorescence signal processing, the key parameter is to find out the initial DNA copies before thermocycling from time history of fluorescence intensity that increases with the PCR temperature cycle. The DNA template concentration within each PCR cycles can be expressed as the following equation:

$c_{i}=\frac{N}{\left(6.02 \times 10^{23}\right)\left(5 \times 10^{-6}\right)} 2^{i}$,

if the PCR efficiency is assumed to be perfect at each cycle. In Eq. (17), $N$ denotes the initial DNA template copies and the unit of DNA concentration is in molar. Substituting Eq. (17) into Eqns. (13) and (16), the relationship of DNA copies and fluorescence intensity can be found. However, fluorescence and excitation light intensity ratio correlated to DNA replication concentration curve equations are exponential functions and explicit terms with respect to discrete fluorescence detection channels and continuous fluorescence wavelength detection optical engine designs. Subsequently two separate signal processing algorithms will be utilized,

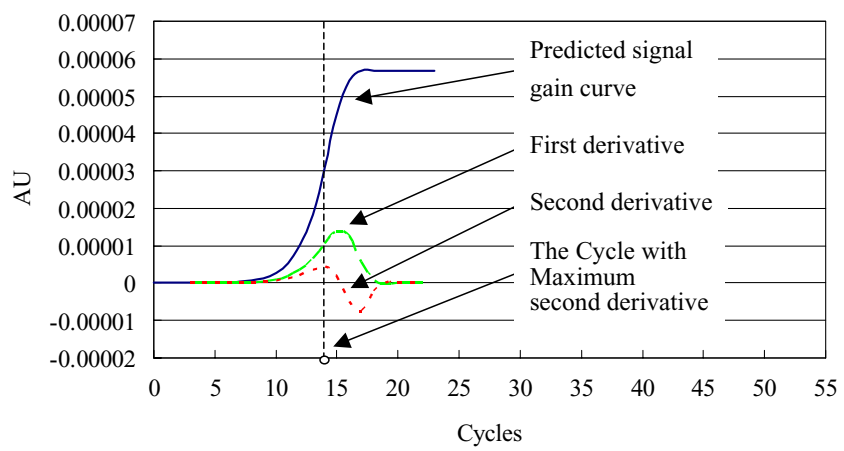

Fig. 9. Predicted signal gain $\left(\Delta F / I_{\lambda}(0)\right)$ at initial DNA copies of $10^{10}$ in $1 \mathrm{ml}$ for the commercial RT-PCR machine, along with its first and second derivatives.

respectively, for the DNA quantification of the commercial machine and of the present prototype.

\subsection{Second derivative maximum method for the commercial RT-PCR machine}

The predicted fluorescence signal gain increases with DNA duplication concentration increment at each PCR cycles. Fig. 9 shows the curves of predicted signal gain, its first and second derivatives of the sample with initial DNA copies of $10^{10}$ in $1 \mathrm{ml}$ for the commercial RT-PCR machine. The first derivative of fluorescence signal gain versus PCR cycle indicates an increasing slope in the early PCR cycles and a decreasing slope as the fluorescence intensity approaches saturation. According to the slope change, a critical cycle $i_{\mathrm{c}}$ can be determined as the second-order derivative reaches the maximum value, as indicated in Fig. 9.

Replacing $a_{i, \lambda} c_{i}+S$ by a parameter $C_{i}$, Eq. (13) becomes

$$
\begin{aligned}
\frac{\Delta F}{I_{\lambda}(0)}= & \text { Const } \frac{C_{i}-S}{C_{i}} \\
& \times\left(-L C_{i}+\frac{1}{2 !} L^{2} C_{i}^{2}-\frac{1}{3 !} L^{3} C_{i}^{3}+\frac{1}{4 !} L^{4} C_{i}^{4}\right) \\
= & -L\left(C_{i}-S\right)+\frac{1}{2 !} L^{2} C_{i}\left(C_{i}-S\right) \\
& -\frac{1}{3 !} L^{3} C_{i}^{2}\left(C_{i}-S\right)+\frac{1}{4 !} L^{4} C_{i}^{3}\left(C_{i}-S\right) .
\end{aligned}
$$

In addition

$C_{i} \propto N 2^{i}$.

As the second derivative of fluorescence intensity at a critical cycle number $i_{\mathrm{c}}$ reaches a maximum value, the third derivative value equals to zero at the same critical cycle number $i_{\mathrm{c}}$. The third derivative of predicted fluorescence signal gain can be expressed as

$$
\begin{aligned}
& \left.\frac{\mathrm{d}^{3}\left(\Delta F / I_{\lambda}(0)\right)}{\mathrm{d} i^{3}}\right|_{i} \\
& =\left.\left[-\left(L^{3}+\frac{1}{4} L^{4} S\right)+L^{4} C_{i}\right]\left(\ln 2 N 2^{i}\right)\right|_{i=i_{\mathrm{c}}}=0 .
\end{aligned}
$$


Eq. (20) equals to zero only if

$-\left(L^{3}+\frac{1}{4} L^{4} S\right)+L^{4} C_{i}=0 \rightarrow C_{i}=\frac{1}{L}+\frac{1}{4} S=$ Const.

Substituting $C_{i}$ from Eq. (19), the relationship between initial DNA copies and the critical cycle number $i_{\mathrm{c}}$ can be expressed as

$N \propto$ Const $\frac{1}{2^{i_{\mathrm{c}}}}=$ Const $\frac{1}{\mathrm{e}^{i_{\mathrm{c}} \ln 2}} \rightarrow \log N \propto \frac{1}{i_{\mathrm{c}}}$.

It indicates that a linear correlation occurs between the log value of initial DNA copies and the reciprocity of the critical cycle $i_{\mathrm{c}}$. From Eq. (22), the experimental sample DNA copies can be interpolated by one set specific concentration in log scale versus the critical cycle plot.

The algorithm is called second derivative maximum method, employed by the commercial PCR machine signal processing. It allows fast calculation and high reproducibility for DNA quantification.

\subsection{Curve fitting method for the prototype}

For the RT-PCR prototype with continuous fluorescence wavelength detection system, the predicted fluorescence signal gain can be expressed by Eq. (16). If all the geometrical data in Eq. (16) are given, Eq. (16) can be reduced as

$\frac{\Delta F}{I_{\lambda}(0)}=2 \pi \phi_{i} a_{i, \lambda} \mathrm{e}^{-L / r} \frac{1}{S}\left(1+\mathrm{e}^{-S L}\right) c_{i}=$ Const $N 2^{i}$.

The first derivative of signal gain is

$\frac{\mathrm{d}\left(\Delta F / I_{\lambda}(0)\right)}{\mathrm{d} i}=$ Const $N \ln 22^{i}$.

The second derivative of signal gain is

$\frac{\mathrm{d}^{2}\left(\Delta F / I_{\lambda}(0)\right)}{\mathrm{d} i^{2}}=$ Const $\times N(\ln 2)^{2} 2^{i}$.

Fig. 10 shows the curves of predicted signal gain, its first derivative and second derivative for the prototype. No critical cycle number $i_{\mathrm{c}}$ can be determined from the maximum

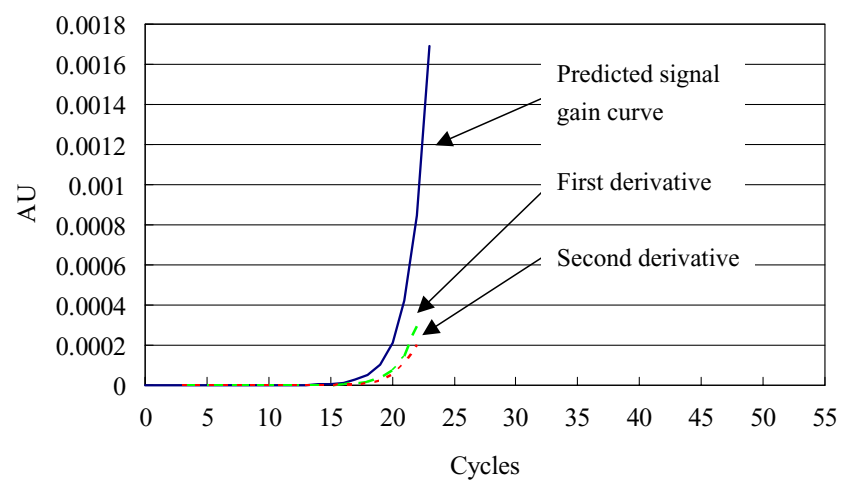

Fig. 10. Predicted signal gain $\left(\Delta F / I_{\lambda}(0)\right)$ at initial DNA copies of $10^{10}$ in $1 \mathrm{ml}$ for the RT-PCR prototype, along with its first and second derivatives. second derivative which is applicable in the predicted signal gain for the commercial RT-PCR machine.

Subsequently a different signal processing method by curve fitting is employed for DNA quantification with RTPCR prototype. The curve fitting method selects one threshold cycle above which fluorescence is above two times the intensity of the standard deviation of the baseline emission. Then six fluorescence readings will be acquired if the fluorescence detection intensity is $1.75-2$ times higher than that of the previous reading. The curves of the fluorescence intensity accumulation in each PCR cycles estimated by Eq. (16) will be fitted to these data and the initial DNA copies can be determined.

The algorithm developed by this study is called curvefitting method. It is more complicated than the second derivative maximum method but the signal processing time only has an imperceptible change since the commercial computer has high-speed computing power.

\section{Results and discussions}

DNA quantification experiments are conducted for DNA samples with four initial DNA copies of $10^{10}, 10^{7}, 10^{5}$, and $10^{3}$. Since the sample is diluted during the preparation of the PCR mix before thermocycling, there lies an uncertainty on the actual number of initial DNA copy. The uncertainty is $1,8,15$, and $20 \%$ in the power index for the tested cases with $10^{10}, 10^{7}, 10^{5}$, and $10^{3}$ specific initial DNA copies, respectively. The fluorescence spectra measured by the RTPCR prototype machine with continuous wavelength detection system for each PCR cycles of the sample with starting $10^{10}$ DNA copies are shown in Fig. 11. The fluorescence emission peak is at $530 \mathrm{~nm}$. The graph shows the minimum

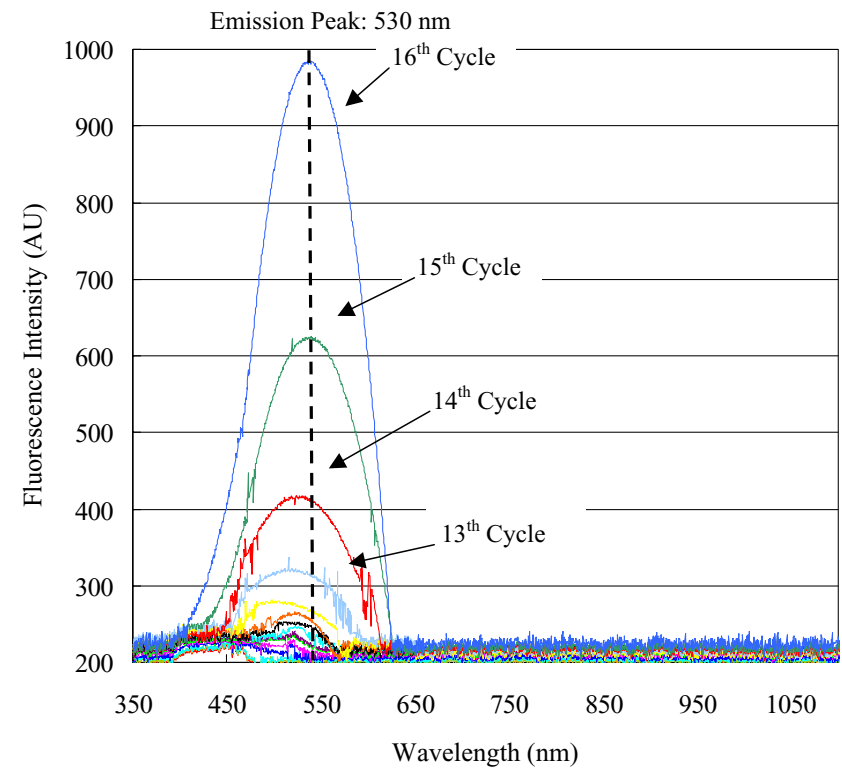

Fig. 11. The spectra of the DNA labeling dye fluorescence within each PCR cycle. 


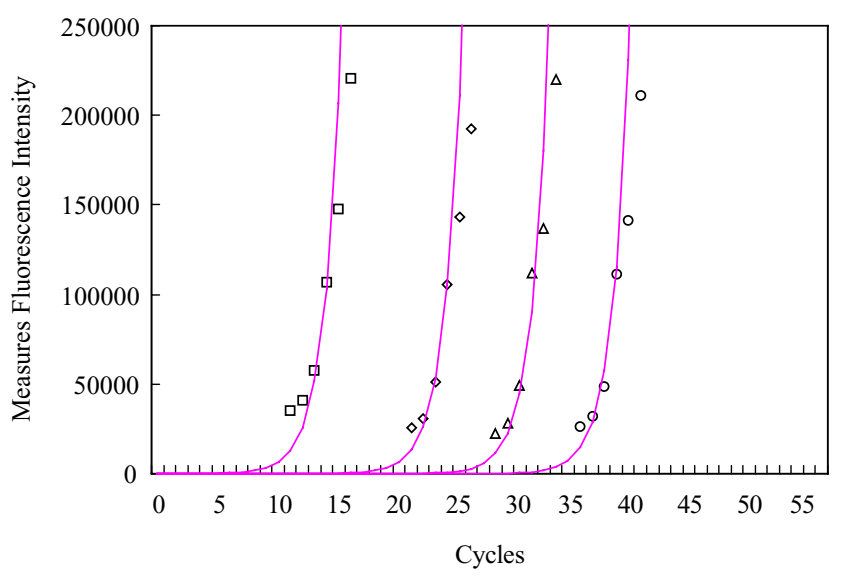

Fig. 12. The DNA quantification results of specific $10^{10}, 10^{7}, 10^{5}$, and $10^{3}$ DNA copies samples. The results by the present prototype with curve fitting method are $1.50 \times 10^{10}, 1.50 \times 10^{7}, 1.15 \times 10^{5}$, and $1.05 \times 10^{3}$.

detection limit with double emission intensity than noise level at 13th PCR cycle. After 16th PCR cycle, the fluorescence intensity reaches the saturation detection limit of the continuous fluorescence wavelength detection system. Although only finite data points are obtained, the curve fitting method needs six data points for DNA quantification.

The fluorescence intensity can be defined by half intensity bandwidth called $3 \mathrm{~dB}$ band integration. Fig. 12 shows the DNA quantification results for PCR mix with four initial DNA copies of $10^{10}, 10^{7}, 10^{5}$, and $10^{3}$. The initial DNA copies obtained by the present prototype with curve fitting method are $1.50 \times 10^{10}, 1.50 \times 10^{7}, 1.15 \times 10^{5}$, and $1.05 \times$ $10^{3}$. The initial DNA copies obtained by the commercial RT-PCR machine with second derivative maximum method are $1.59 \times 10^{10}, 3.99 \times 10^{6}, 2.27 \times 10^{4}$, and $9.60 \times 10^{3}$.

The two curves in Fig. 13 show comparison of the quantification results obtained by the commercial RT-PCR machine and the prototype. The curves express the specific initial DNA copies and the real measurement results in
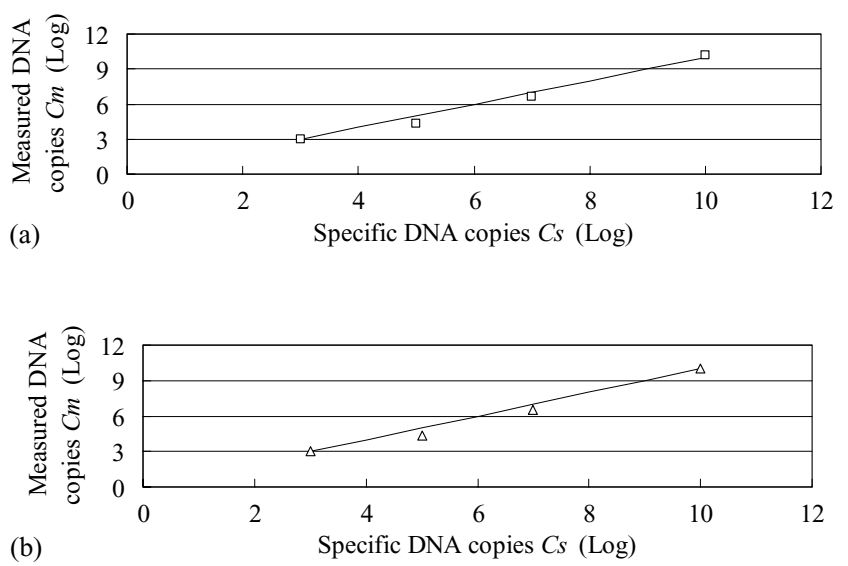

Fig. 13. DNA quantification curves express the specified initial DNA copies and the real measurement results in log and log scale. (a) Commercial RT-PCR machine: $R^{2}=0.988$. (b) Present prototype machine: $R^{2}=0.998$.

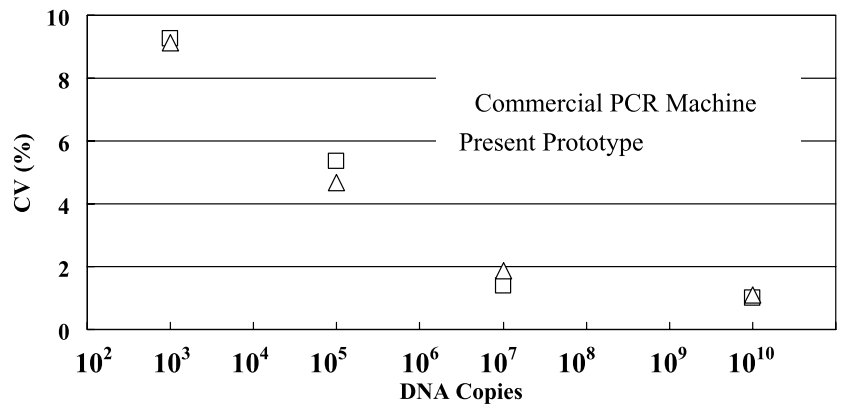

Fig. 14. The reproducibility of the RT-PCR machine tested by intra-assay with three replicates for different initial DNA copies in the present prototype and in the commercial RT-PCR machine.

$\log$ and $\log$ scale. The $R^{2}$ values indicate the accuracy of the measurement results. As it approaches 1, the accuracy approaches perfection. The value can be determined by

$R^{2}=\frac{n\left(\sum C_{\mathrm{s}} C_{\mathrm{m}}\right)-\left(\sum C_{\mathrm{s}}\right)\left(\sum C_{\mathrm{m}}\right)}{\left[n \sum C_{\mathrm{s}}^{2}-\left(\sum C_{\mathrm{s}}\right)^{2}\right]\left[n\left(\sum C_{\mathrm{m}}^{2}\right)-\left(\sum C_{\mathrm{s}}\right)^{2}\right]}$.

where $C_{\mathrm{s}}$ means the specified initial DNA copies and $C_{\mathrm{m}}$ means the measured initial DNA copies, both can be referred to as the $X$ - and $Y$-axis in Fig. 13. The $R^{2}$ values for the present prototype and commercial RT-PCR machine are 0.998 and 0.988 , respectively.

The reproducibility of the RT-PCR machine depends on such factors as the fluorescence detection system accuracy and the thermal cycling induced PCR efficiency discrepancy. Coefficient of variation (CV) with a unit in percentage is usually employed for the index and it is defined by

$\mathrm{CV}=\frac{\log _{10}\left(\sum C_{\mathrm{m}} / n\right)-\log _{10}\left(C_{\mathrm{s}}\right)}{\log _{10}\left(C_{\mathrm{s}}\right)} \times 100 \%$.

The CV value indicates discrepancy between the average of several quantification results and the specified DNA copies value. A low $\mathrm{CV}$ value means the system has high reproducibility and all factors induced measurement errors are minimized. Fig. 14 shows the $\mathrm{CV}$ value tested by intra-assay with three replicates for different initial DNA copies in the present system and in the commercial RT-PCR system. As shown in Fig. 14, the reproducibility of the present prototype is similar to that of the commercial RT-PCR machine.

\section{Conclusions}

The RT-PCR prototype machine with a miniature spectrometer for continuous fluorescence wavelength sensing was built for DNA quantification in a micro liter volume glass capillary. Different fluorescence sensing system designs were analyzed by analytical model. A signal processing algorithm called curve fitting method was developed based on the model.

It was proved by HBV SC11 DNA template that the present prototype with the curve fitting method signal 
processing algorithm could provide the same accuracy and reproducibility of DNA quantification as compared to the commercial PCR machine. Additionally, the system can provide wide spectral spans from 350 to $1100 \mathrm{~nm}$ for multiplex DNA quantification. However, as fluorescence intensity has to be determined by fluorescence $3 \mathrm{~dB}$ band integration, when performing the DNA quantification experiments, we must consider the fluorescence wavelength span of the labeling dye for these dyes to be nicely separated spectrally for color multiplexing. SYBR Green I dye with $521 \mathrm{~nm}$ emission peak, CY3 with $565 \mathrm{~nm}$ emission peak, LC Red 640 with $640 \mathrm{~nm}$ emission peak, CY5 with $670 \mathrm{~nm}$ emission peak, LC Red 705 with $705 \mathrm{~nm}$ emission peak and IR Dye 800 with 812 emission peak will be good choices for multiplex DNA quantification and the experiment will be performed in the next stage.

By the miniature spectrometer, the RT-PCR prototype has been set up successfully. The system has the same accuracy and reproducibility as the commercial RT-PCR machine and it can provide wide spectral fluorescence sensing capability for multiplex DNA quantification without the detection channels limitation of the commercial PCR machine. Rapid DNA amplification combined with the spectral fluorescence sensing system proposed by this study will be practically useful for methods development and diagnostics.

\section{Acknowledgements}

This work was financially supported by research project No. 90-2212-F002-197 of the National Science Council, Taiwan, ROC.

\section{References}

[1] K.B. Mullis, F. Ferre, R.A. Gibbs, The Polymerase Chain Reaction, Birkhauser, Boston, 1994.
[2] C.T. Wittwer, M.G. Herrmann, A.A. Moss, R.P. Rasmussen, Continuous fluorescence monitoring of rapid cycle DNA amplification, BioTechniques 22 (1997) 130-138.

[3] C.T. Wittwer, K.M. Ririe, R.V. Andrew, D.A. David, R.A. Gundry, U.J. Balis, The LightCycler ${ }^{\mathrm{TM}}$ : a microvolume multisample fluorimeter with rapid temperature control, BioTechniques 22 (1997) 176181.

[4] http://www.appliedbiosystems.com/support/tutorials/pdf/rtpcr_vs_ tradpcr.pdf.

[5] C.T. Wittwer, M.G. Herrmann, C.N. Gundry, K.S.J. Elenitobe-Johnson, Real-time multiplex PCR assays, Methods 25 (2001) 430-442.

[6] http://www.OceanOptics.COM/Technical/GratingCharts.asp.

[7] http://omlc.ogi.edu/spectra/water/data/sogandares97.dat.

[8] http://elchem.kaist.ac.kr/vt/chem-ed/spec/beerslaw.htm.

\section{Biographies}

Da-Sheng Lee received his Master degree in mechanical engineering from the Nation Taiwan University, Taipei, Taiwan ROC, in 1996. Since he has joined ITRI, Hsinchu, Taiwan, ROC, in 1996, he has been serving as a vice researcher of ERL and laboratory leader of Sound and Vibration laboratory. $\mathrm{He}$ is currently pursuing the $\mathrm{PhD}$ degree in the same school. His current research interests include the Lab on a chip for biology and real-time PCR system for DNA quantitative sequencing.

Meng-Hsun Wu received the BS degree in Mechanical Engineering from the National Taiwan University, Taiwan, ROC, in 2002. He is currently pursuing the MS degree at National Taiwan University, Taipei, Taiwan. His current research interests include MEMS fabrication, real-time PCR system for DNA quantitative Sequencing.

U. Ramesh completed his B Tech degree in Mechanical Engineering, JNT University, India. Now he is doing masters in fluid mechanics in National Taiwan Univeristy, Taiwan.

Ping-Hei Chen received his bachelor degree from National Taiwan University in 1980 and PhD degree from University of Minnesota in 1988. Afterwards, he joined the Department of Mechanical Engineering of National Taiwan University as an associate professor. He was promoted to full professor in 1996. He had served as the chairman of the Department of Mechanical Engineering of National Taiwan University from 1998 to 2001. His research areas include micro-thermal-fluid systems, Lab-on-achip for DNA detections, Nanofluid, MEMS fabrication technology, and cooling devices for electronic equipments. 